\title{
Some Results on Number Theory and Differential Equations
}

\author{
B. M. Cerna Maguiña ${ }^{1, *}$, Dik D. Lujerio Garcia ${ }^{1}$, Héctor F. Maguiña ${ }^{2}$ \\ ${ }^{1}$ Academic Department of Mathematics, Science Faculty, Santiago Antúnez de Mayolo National University, Shancayan Campus, Perú \\ ${ }^{2}$ National University of San Marcos, Street Germán Amézaga N³75. Pabellón "F” -Accounting sciences, University City, Perú \\ * Corresponding Author: bcernam@unasam.edu.pe,dlujeriog@unasam.edu.pe, hcernam@unmsm.edu.pe
}

Received March 10, 2021; Revised May 24, 2021; Accepted June 6, 2021

Cite This Paper in the following Citation Styles

(a): [1] B. M. Cerna Maguiña, Dik D. Lujerio Garcia, Héctor F. Maguiña, "Some Results on Number Theory and Differential Equations," Mathematics and Statistics, Vol.9, No.6, pp. 984-993, 2021. DOI: 10.13189/ms.2021.090614

(b): B. M. Cerna Maguiña, Dik D. Lujerio Garcia, Héctor F. Maguiña, (2021). Some Results on Number Theory and Differential Equations. Mathematics and Statistics, 9(6), 984-993. DOI: 10.13189/ms.2021.090614

Copyright $@ 2021$ by authors, all rights reserved. Authors agree that this article remains permanently open access under the terms of the Creative Commons Attribution License 4.0 International License

\begin{abstract}
In this work, using the basic tools of functional analysis, we obtain a technique that allows us to obtain important results, related to quadratic equations in two variables that represent a natural number and differential equations. We show the possible ways to write an even number that ends in six, as the sum of two odd numbers and we establish conditions for said odd numbers to be prime, also making use of a suitable linear functional $F: \mathbb{R}^{3} \rightarrow \mathbb{R}$ we obtain representations of natural numbers of the form $(10 A+9), A \in \mathbb{N}$ in order to obtain positive integer solutions of the equation quadratic $(10 x+9)(10 y+9)=P$ where $P$ is a natural number given that it ends with one. And finally, we show with three examples the use of the proposed technique to solve some ordinary and partial linear differential equations. We believe that the third corollary of our first result of this investigation can help to demonstrate the strong Goldbach conjecture.
\end{abstract}

Keywords Prime Numbers, Diophantine Equations, Differential Equations, Number Theory, Functional Analysis

\section{Introduction}

In [4], the author shows an application of Riez's theorem to show the existence and uniqueness of the generalized solution of the Poisson equation in the plane. Using the technique described in [1], we obtain the relation (47), which is easier to solve. In chapter VI he addresses the problem of eigenvalues and by means of Theorem [6.3.1] shows its application to the study of the membrane. We, similarly to the previous example, obtain the equation (48). In [3] Pag. 167, the author proposes to solve the ordinary differential equation of the second order by means of a uniform network using the difference scheme. In [1] a technique based on the use of linear functionals was proposed to help solve problems related to number theory. In [5] we obtained bounds for integer solutions of quadratic polynomials in two variables that represent a given natural number. The objective of this work was to use the same technique to tackle other problems related to the theory of numbers and differential equations. The methodology used was deductive and inductive. The contribution of this work is to define special linear functions, in the plane or in three-dimensional space, for the study of quadratic equations in two variables that represent a natural number and the resolution of some differential equations.

The following work is divided into two sections, in the first section we show some results of Number Theory. And in the second section we show some results related to ordinary and partial linear differential equations. 


\section{Some results on number theory}

In the following Theorem, we only address the cases of natural numbers that end in six, which are obtained, via the sum of two odd numbers.

Theorem 2.1. Let $M$ be an even number endig in 6 , then there exist $a, b \in \mathbb{N}$, where $a$ is divisor of $M$, and a and $b$ are relatively primes with $a>b$ and $M \frac{(a+b)}{2 a} \in \mathbb{N}, M \frac{(a-b)}{2 a} \in \mathbb{N}$ such that: $M=\frac{M(a+b)}{2 a}+\frac{M(a-b)}{2 a}$.

Proof. We observe that there are three possible cases.

Caso 1. Let

$$
M=(K-\alpha)+(K+\alpha)=\left(10 k_{1}+3\right)+\left(10 k_{2}+3\right)
$$

where $k_{2}=\frac{K-3+\alpha}{10}, k_{1}=\frac{K-3-\alpha}{10}, \alpha, k_{1}, k_{2}$ are natural numbers.

Let us define the next linear functional

$$
F(x, y)=\left(10 k_{1}+3\right) x+\left(10 k_{2}+3\right) y .
$$

So, $\operatorname{ker} F=\left\{\left(-\left(10 k_{2}+3\right), 10 k_{1}+3\right)\right\},\{\operatorname{ker} F\}^{\perp}=\left\{\left(10 k_{1}+3,10 k_{2}+3\right)\right\}$. We have

$$
F(1,1)=M \text {. }
$$

Moreover

$$
(1,1)=\lambda_{1}\left(-\left(10 k_{2}+3\right),\left(10 k_{1}+3\right)\right)+\lambda_{2}\left(\left(10 k_{1}+3\right), 10 k_{2}+3\right) .
$$

From (2) and (4) we have

$$
M=\lambda_{2}\left[\left(10 k_{1}+3\right)^{2}+\left(10 k_{2}+3\right)^{2}\right] .
$$

From (5) and (1) it follows

$$
M=\lambda_{2}\left[(K-\alpha)^{2}+(K+\alpha)^{2}\right]=\lambda_{2}\left[2 K^{2}+2 \alpha^{2}\right]
$$

from (5) it follows that $\lambda_{2} \in \mathbb{Q}$. Let $\lambda_{2}=\frac{m}{n}$, where $m$ and $n$ are relatively primes, $m<n$. From this last relation one has

$$
|M m-n|=\sqrt{n^{2}-4 \alpha^{2} m^{2}} .
$$

Using the Fermat's Theorem for the power 2 one has $n=a^{2}+b^{2}, w=a^{2}-b^{2}, a>b, \alpha m=a b$; then, using these ralationships into (6) one has

$$
\alpha=\frac{M b}{2 a} \text { or } \alpha=\frac{M a}{2 b}
$$

From (2) one has

$$
F(1,0)=10 k_{1}+3
$$

From (8) we see that $10 k_{1}+3$ is a number ending in 3 . Let us assume that this number in not prime. Then

$$
10 k_{1}+3=(10 A+7)(10 B+9) \text { or } 10 k_{1}+3=(10 A+3)(10 B+1)
$$

where $(A, B) \in \mathbb{N} \times \mathbb{N}$.

So, one has

$$
(1,0)=r_{1}\left(-\left(10 k_{2}+3\right),\left(10 k_{1}+3\right)\right)+r_{2}\left(10 k_{1}+3,10 k_{2}+3\right),
$$

from (8) y (10)

$$
10 k_{1}+3=r_{2}\left(\left(10 k_{1}+3\right)^{2}+\left(10 k_{2}+3\right)^{2}\right) .
$$


From the relation (2) one has

$$
F(0,1)=10 k_{2}+3
$$

From (12) one has $10 k_{2}+3$ is a number ending in 3; let us assume that this in not a prime number. Then

$$
10 k_{2}+3=(10 C+3)(10 D+1) \text { or } 10 k_{2}+3=(10 C+7)(10 D+9)
$$

where $(C, D) \in \mathbb{N} \times \mathbb{N}$.

From the relation (12) and the relationship

$(0,1)=t_{1}\left(-\left(10 k_{2}+3\right),\left(10 k_{1}+3\right)\right)+t_{2}\left(10 k_{1}+3,10 k_{2}+3\right)$, one gets

$$
10 k_{2}+3=t_{2}\left[\left(10 k_{1}+3\right)^{2}+\left(10 k_{2}+3\right)^{2}\right] .
$$

Since (1) $10 k_{1}+3=K-\alpha$ y $10 k_{2}+3=K+\alpha$, from (11) and (12) one has

$$
\begin{aligned}
M & =\left(r_{2}+t_{2}\right)\left[\left(10 k_{1}+3\right)^{2}+\left(10 k_{2}+3\right)^{2}\right]=\left(r_{2}+t_{2}\right)\left[\frac{M^{2}}{2}+2 \alpha^{2}\right] \\
2 \alpha & =\left(t_{2}-r_{2}\right)\left[\left(10 k_{1}+3\right)^{2}+\left(10 k_{2}+3\right)^{2}\right]=\left(t_{2}-r_{2}\right)\left[\frac{M^{2}}{2}+2 \alpha^{2}\right]
\end{aligned}
$$

From (15) and (7) one can get the following

$$
t_{2}=\frac{a(a+b)}{M\left(a^{2}+b^{2}\right)} \text { and } r_{2}=\frac{a(a-b)}{M\left(a^{2}+b^{2}\right)}, a>b .
$$

From the relations (11), (16) and (14) one gets

$$
10 k_{1}+3=\frac{(a-b) M}{2 a} \text { and } 10 k_{2}+3=\frac{(a+b) M}{2 a}, a>b .
$$

where $k_{1}=\frac{M(a-b)-6 a}{20 a}$ and $k_{2}=\frac{M(a+b)-6 a}{20 a}$.

Caso 2. The other possibility to write $M$ is given by the next equation

$$
M=(K-\alpha)+(K+\alpha)=\left(10 k_{1}+9\right)+\left(10 k_{2}+7\right)=\left(10 k_{1}+9\right)+\left(10 k_{2}+7\right)
$$

The process is similar to (1); so, $k_{1}, k_{2}$ are given by

$k_{1}=\frac{K-9-\alpha}{10}, k_{2}=\frac{K+\alpha-7}{10}$ o $k_{2}=\frac{K-7-\alpha}{10}, k_{1}=\frac{K+\alpha-9}{10}$ where $\alpha=\frac{M b}{2 a}, 2 K=M$.

Caso 3. Finally, the last possibility to write $M$ is in the following form

$$
M=5+\left(10 k_{1}+1\right)=(K-\alpha)+(K+\alpha)
$$

where $\alpha=K-5$ y $k_{1}=\frac{K-3}{5}$.

Corollary 2.1. $\left(10 k_{1}+3\right)$ and $\left(10 k_{2}+3\right)$ are primes if and only if $(a-b)$ and $(a+b)$ are prime numbers. This happens when $2 a=M$.

Proof. The proof follows by using (17).

Corollary 2.2. The relationships between $\alpha, m, n$ and $K$ are given by $m=K$ and $\alpha=\sqrt{n-K^{2}}$, where $K^{2} \leq n \leq 2 K^{2}$.

Proof. Use the relation (6). For $K$ even, $\alpha$ is odd and $n$ is odd. For $K$ odd, $\alpha$ is even and $n$ is odd.

Remark 2.1. The primality of a natural number is subject to the integer solution of the equation $(x-n)^{2}+(y-n)^{2}=2 n^{2}$, where $n \in \mathbb{N}$.

Corollary 2.3. Let $M=2 K$, where $M$ is a natural number ending in 6. For each $K$ being a natural number ending in 8 we hava that if $K+\alpha$ and $K-\alpha$ are prime numbers then $\alpha \in\{1,11,21, \ldots, K-7\}$ or $\{5,15,25, \ldots, K-3\}$. If $K$ ends in 3 then $\alpha \in\{0,10,20, \ldots, K-3\}$ or $\alpha \in\{6,16,26, \ldots, K-7\}$. 
Proof. Simply, we use the equations (17) y (18) to obtain $\frac{(K+\alpha-9)^{2}}{50} \in \mathbb{N}$ or $\frac{(K+\alpha-3)^{2}}{50} \in \mathbb{N}, 0 \leq \alpha \leq K$, and from this the result follows.

Remark 2.2. Let $K+\alpha_{0}$ and $K-\alpha_{0}$ be prime numbers satisfying the condition $M=2 K, K$ ending in 8 and $\alpha_{0} \in$ $\{1,11, \ldots, K-7\}$.

For $\widetilde{M}=M+10=2 \widetilde{K}$ imply $\widetilde{K}=K+5, \widetilde{\alpha}_{0}=\alpha_{0}-1 y\left(\widetilde{K}+\widetilde{\alpha}_{0}\right)+\left(\widetilde{K}-\widetilde{\alpha}_{0}\right)=2 \widetilde{K}=\widetilde{M}$, where $\frac{\left(K+\alpha_{0}+1\right)^{2}}{50} \in \mathbb{N}$.

If $K$ ends in 8 and $\alpha_{0} \in\{5,15, \ldots, K-3\}$ one can have $\widetilde{K}=K+5, \widetilde{\alpha}_{0}=\alpha_{0}+1$ y $\left(\widetilde{K}+\widetilde{\alpha}_{0}\right)+\left(\widetilde{K}-\widetilde{\alpha}_{0}\right)=2 \widetilde{K}=\widetilde{M}$, where $\frac{\left(K+\alpha_{0}-3\right)^{2}}{50} \in \mathbb{N}$. A similar result can be obtained if $K$ ends in 3 .

Example 2.1. We use Corollary 2.3 and observation 2.2, which are supported by Theorem 2.1. Let $M=416$, then $K=208$, $K$ ends in 8 . If $K+\alpha$ and $K-\alpha$ are prime numbers then there is

$$
\alpha \in\{1,11,21, \ldots, 201\} \quad \text { or } \quad\{5,15,25, \ldots, 205\}
$$

Thus,

\begin{tabular}{|c|c|}
\hline \multicolumn{2}{|c|}{ Tabla 1} \\
\hline$K+\alpha$ & $K-\alpha$ \\
\hline 209 & 207 \\
219 & 197 \\
229 & 187 \\
239 & 177 \\
249 & 167 \\
259 & 157 \\
269 & 147 \\
279 & 137 \\
289 & 127 \\
299 & 117 \\
309 & 107 \\
319 & 97 \\
329 & 87 \\
339 & 77 \\
349 & 67 \\
359 & 57 \\
369 & 47 \\
379 & 37 \\
389 & 27 \\
399 & 17 \\
409 & 7 \\
\hline
\end{tabular}

\begin{tabular}{|c|c|}
\hline \multicolumn{2}{|c|}{ Tabla 2} \\
\hline$K+\alpha$ & $K-\alpha$ \\
\hline 213 & 203 \\
223 & 193 \\
233 & 183 \\
243 & 173 \\
253 & 163 \\
263 & 153 \\
273 & 143 \\
283 & 133 \\
293 & 123 \\
303 & 113 \\
313 & 103 \\
323 & 93 \\
333 & 83 \\
343 & 73 \\
353 & 63 \\
363 & 53 \\
373 & 43 \\
383 & 33 \\
393 & 23 \\
403 & 13 \\
413 & 3 \\
\hline
\end{tabular}

From the first table the possible primes are given by $(219,197),(229,187),(249,167),(279,137),(289,127)$, (309, 107), $(319,97),(339,77),(349,67),(369,47),(379,37),(399,17),(409,7)$. From the second table the possible primes are given by $(213,203),(223,193),(243,173),(253,163),(273,143),(283,133),(303,113),(313,103),(333,83),(343,73),(363,53)$, (373, 43), (393, 23), (403, 13). That is, in the first and second tables, the numbers that are multiples of three were eliminated, since these numbers are not prime.

Then $M+10=426=2 \widetilde{K}$ and consequently $\widetilde{K}=213, \widetilde{K}$ ends in three. If $\widetilde{K}+\widetilde{\alpha}$ and $\widetilde{K}-\widetilde{\alpha}$ are prime numbers, then it exists $\widetilde{\alpha}$ such that:

$$
\widetilde{\alpha} \in\{0,10,20, \ldots, 210\} \quad \text { or } \quad\{6,16,26, \ldots, 206\}
$$

Thus, 


\begin{tabular}{|c|c|}
\hline \multicolumn{2}{|c|}{ Tabla 3} \\
\hline$\widetilde{K}+\widetilde{\alpha}$ & $\widetilde{K}-\widetilde{\alpha}$ \\
\hline 213 & 213 \\
223 & 203 \\
233 & 193 \\
243 & 183 \\
253 & 173 \\
263 & 163 \\
273 & 153 \\
283 & 143 \\
293 & 133 \\
303 & 123 \\
313 & 113 \\
333 & 93 \\
343 & 83 \\
353 & 73 \\
363 & 63 \\
373 & 53 \\
383 & 43 \\
393 & 33 \\
403 & 23 \\
413 & 13 \\
423 & 3 \\
\hline
\end{tabular}

\begin{tabular}{|c|c|}
\hline \multicolumn{2}{|c|}{ Tabla 4} \\
\hline$\widetilde{K}+\widetilde{\alpha}$ & $\widetilde{K}-\widetilde{\alpha}$ \\
\hline 219 & 207 \\
229 & 197 \\
239 & 187 \\
249 & 177 \\
259 & 167 \\
269 & 157 \\
279 & 147 \\
289 & 137 \\
299 & 127 \\
309 & 117 \\
319 & 107 \\
329 & 97 \\
339 & 87 \\
349 & 77 \\
359 & 67 \\
369 & 57 \\
379 & 47 \\
389 & 37 \\
399 & 27 \\
409 & 17 \\
419 & 7 \\
\hline &
\end{tabular}

Similar to the previous table we have the possible primes for the first table: (223, 203), (233, 193), (253, 173), (263, 163), (283, 143), (293, 133), (313, 113), (323, 103), (343, 83), (353,73), (373, 53), (383, 43), (403, 23), (413, 13).

For the second table we have: (229, 197), (239, 187), (259, 167), (269, 157), (289, 137), (299, 127), (319, 107), (329, 97), (349,77), (359,67), (389,37), (409, 17), (419, 7).

In the pair $(379,37)$ they are both prime. Therefore it is clear that this occurs for $\alpha_{0}=171$. Thus $\widetilde{\alpha}_{0}=\alpha_{0}-1=170$, $\widetilde{K}=K+5$. Thus $\widetilde{K}+\widetilde{\alpha}_{0}=373$ and $\widetilde{K}-\widetilde{\alpha}_{0}=43$, and in the pair $(373,43)$ both are prime numbers. Similar process for $\alpha \in\{6,16,26, \ldots, 206\}$.

The last possibility to write $M=416$ as the sum of two possible primes is $416=5+411$, but 411 is not prime.

Then $416+10=426=5+421$, in the pair $(5,421)$ both are prime. For this last process it is not possible to apply the induction method.

The induction process can be carried out in Table (1) with table (3) or Table (2) with Table (4). That is, if we suppose that there exists $\alpha_{0}$ that belongs to Table (1) such that $K+\alpha_{0}$ and $K-\alpha_{0}$ are primes, then the question is: Will there be $\widetilde{\alpha}_{0}$ qthat belongs to Table (3) such that $\widetilde{K}+\widetilde{\alpha}_{0}=K+5+\alpha_{0}-1=K+4+\alpha_{0}$ and $\widetilde{K}-\widetilde{\alpha}_{0}=K+5-\left(\alpha_{0}-1\right)=K+6-\alpha_{0}$ are prime numbers?. Similar inductive process if $\alpha_{0}$ belongs to Table (2).

Remark 2.3. If $K-\alpha$ and $K+\alpha$ end in 3 where $M=2 K$, then $K-\alpha$ and $K+\alpha$ possess the represntations given in (9) and (13), and if they possess integer solutions using the Remark 2.6 we have

$$
\frac{\sqrt{10 k_{1}+3}}{37.3}-\frac{7}{10} \leq A \leq \frac{\sqrt{10 k_{1}+3}}{10}-\frac{7}{10}
$$

or

$$
\frac{\sqrt{10 k_{1}+3}}{37.3}-\frac{3}{10} \leq A \leq \frac{\sqrt{10 k_{1}+3}}{10}-\frac{3}{10}, A<B
$$

This is for the first representation of $K-\alpha$ given in (9).

We can obtain similar results if $K+\alpha$ has the possible representation given in (13).

Theorem 2.2. Let $P=(10 A+9)(10 B+9),(A, B) \in \mathbb{N} \times \mathbb{N}$. There is $K \in\langle 1, \sqrt{2}\rangle$ such that:

$$
10 A+9=P^{\frac{1}{2}-\frac{\sqrt{2-K^{2}}}{2 K}}
$$

$\operatorname{con} A<B$.

Proof. Let's define $F(x, y, z)=[\ln (10 A+9)] x+[\ln (10 B+9)] y+[\ln P] z$. Thus

$$
F(1,1,1)=\ln (10 A+9)+\ln (10 B+9)+\ln P
$$


Furthermore

$$
\begin{aligned}
\operatorname{Ker} F & =\{(-\ln P, 0, \ln (10 A+9)),(-\ln (10 B+9), \ln (10 A+9), 0)\} \\
\operatorname{Ker} F^{\perp} & =\{(\ln (10 A+9), \ln (10 B+9), \ln P)\}
\end{aligned}
$$

Since

$$
\begin{aligned}
(1,1,1)= & \lambda_{1}(-\ln P, 0, \ln (10 A+9))+\lambda_{2}(-\ln (10 B+9), \ln (10 A+9), 0)+ \\
& \lambda_{3}(\ln (10 A+9), \ln (10 B+9), \ln P)
\end{aligned}
$$

From (20) and this last relation we have:

$$
2 \ln P=\lambda_{3}\left[\ln ^{2}(10 A+9)+\ln ^{2}(10 B+9)+\ln ^{2} P\right]
$$

From the relation (21) we obtain:

$$
\left\{\begin{array}{l}
\ln (10 A+9)=\frac{1}{\lambda_{3}} \sin \Phi \cos \theta \\
\ln (10 B+9)=\frac{1}{\lambda_{3}} \sin \Phi \sin \theta \\
\ln P=\frac{1}{\lambda_{3}}(\cos \Phi+1)
\end{array}\right.
$$

From the relation (22) we obtain

$$
\cos \theta+\sin \theta=\frac{\cos \Phi+1}{\sin \Phi}=K
$$

It is clear that $\theta=\theta(A, B, C), \Phi=\Phi(A, B, P), \lambda_{3}=\lambda_{3}(A, B, P), K=K(A, B, P), \theta \in\left\langle 0, \frac{\pi}{2}\right\rangle, \Phi \in\langle 0, \pi\rangle, 1<K<\sqrt{2}$.

From (23) we get $\sin \theta=\sqrt{\frac{1-K \sqrt{2-K^{2}}}{2}}, \cos \theta=\sqrt{\frac{1+K \sqrt{2-K^{2}}}{2}}, \cos \Phi=\frac{K^{2}-1}{K^{2}+1}, \sin \Phi=\frac{2 K}{K^{2}+1}$.

Also from ((22)) we obtain

$$
\frac{10 B+9}{10 A+9}=P^{\frac{\sin \Phi}{\cos \Phi+1}(\cos \theta-\sin \theta)}
$$

Using the values of $\cos \theta$ and $\sin \theta$ and the relation (23) we obtain in (24) the following:

$$
\frac{(10 B+9)}{(10 A+9)}=P^{\frac{\sqrt{2-K 2}}{K}}
$$

From the relation (25) we have that for $A<B$ and $P=(10 A+9)(10 B+9)$ the following

$$
(10 A+9)=P^{\frac{1}{2}-\frac{\sqrt{2-K^{2}}}{2 K}}
$$

Corollary 2.4. For, $0<a=\frac{\sqrt{2-K^{2}}}{2 K}<\frac{1}{2}$ we have

$$
1<P^{\frac{a}{2}}<\frac{\sqrt{6}+\sqrt{2}}{2} .
$$

Proof. We use Theorem (2.1) of [5] in which $\frac{\sqrt{P}-9}{5} \leq A+B \leq \frac{2}{5}(\sqrt{P}-9)$, and using this relation together with the value of

$$
A=\frac{P^{1 / 2-a}-9}{10}, \quad B=\frac{P^{1 / 2+a}-9}{10}
$$

, we get the required value.

Remark 2.4. Similar results are obtained for $P=(10 A+1)(10 B+1), P=(10 A+7)(10 B+3)$, with $(A, B) \in \mathbb{N} \times \mathbb{N}$.

Remark 2.5. If we want a smaller bound for a we can use:

$$
P^{\frac{a}{2}} \leq \frac{\sqrt{2-\frac{18}{\sqrt{p}}}+\sqrt{6-\frac{18}{\sqrt{p}}}}{2}
$$

Remark 2.6. $1 \leq \frac{10 B+9}{10 A+9} \leq 13.96, A<B, A, B \in \mathbb{N} \times \mathbb{N}$. 


\section{Some results on differential equations}

We show below that with the technique proposed in [1], we can also solve equations such as:

Example 3.1. [3] Let us consider the Cauchy problem in the interval [0, $\ell]$

$$
\frac{d^{2} x}{d t^{2}}+b(t) \frac{d x}{d t}+c(t) x(t)=y(t), x(0)=\alpha, x^{\prime}(0)=\beta .
$$

It is convenient to write it in the form of the system $\frac{d x}{d t}-u(t)=0$,

$$
\frac{d u}{d t}+b(t) u(t)+c(t) x(t)=y(t), x(0)=\alpha, u(0)=\beta .
$$

In order to solve (26) let us apply the Theorem (2.1).

Let $F(x, y)=u^{\prime}(t) x+b(t) u(t) y$, and assume that $\left|u^{\prime}(t)\right| \leq M_{1},|b(t)| \leq M_{2},|u(t)| \leq M_{3} \forall t \in[0, \ell]$. Then the operator $F$ is bounded. Then ker $F$ is closed. (Even though this is not necessary since every linear operator $F: \mathbb{R}^{n} \longrightarrow \mathbb{R}^{m}$ is bounded).

So, one has $\operatorname{ker} F=\left\{\left(-b(t) u(t), u^{\prime}(t)\right)\right\},\{\operatorname{ker} F\}^{\perp}=\left\{\left(u^{\prime}(t),-b(t) u(t)\right)\right\}$

$$
F(1,1)=u^{\prime}(t)+b(t) u(t)=f(t), \text { where } f(t)=y(t)-c(t) x(t)
$$

Since

$$
(1,1)=\lambda_{1}\left(-b(t) u(t), u^{\prime}(t)\right)+\lambda_{2}\left(u^{\prime}(t), b(t) u(t)\right),
$$

then

$$
f(t)=\lambda_{2}\left(u^{\prime 2}(t)+b^{2}(t) u^{2}(t)\right)
$$

A parametrization satisfying (29) is

$$
\begin{aligned}
u^{\prime}(t) & =\sqrt{\frac{f(t)}{\lambda_{2}(t)}} \sin \left(\frac{\pi t}{\ell}\right) \\
b(t) u(t) & =\sqrt{\frac{f(t)}{\lambda_{2}(t)}} \cos \left(\frac{\pi t}{\ell}\right) .
\end{aligned}
$$

From the relations (30) and (31) one gets

$$
\lambda_{2}(t)=\frac{1+\sin 2\left(\frac{\pi t}{\ell}\right)}{f(t)}
$$

From the relations (28) one gets

$$
\lambda_{2}(t)=\frac{f(t)}{u^{\prime 2}(t)+u^{2}(t) b^{2}(t)}
$$

From (32) and (33) one has

$$
\left[u^{\prime 2}(t)+u^{2}(t) b^{2}(t)\right]\left(1+\sin 2\left(\frac{\pi t}{\ell}\right)\right)=f^{2}(t)
$$

From (30) and (31) one has

$$
\frac{u^{\prime}(t)}{u(t)}=b(t) \tan \left(\frac{\pi t}{\ell}\right)
$$

From (34) and (35) one has

$$
f(t)=u(t) b(t)\left(1+\tan \left(\frac{\pi t}{\ell}\right)\right)
$$

Therefore from (35) one gets that $x(t)=\alpha+\beta \int_{0}^{t} e^{G(t)} d \tau$, where $G(t)=\int_{0}^{\tau} b(t) \tan \left(\frac{\pi t}{\ell}\right) d t$, and in addition from (36) one has that

$$
y(t)-\beta e^{G(t)}\left(1+\tan \left(\frac{\pi t}{\ell}\right)\right) b(t)=\left(\alpha+\beta \int_{0}^{t} e^{G(t)} d \tau\right) c(t)
$$


Remark 3.1. As a particular case, let us choose $\tan \left(\frac{\pi t}{l}\right)=1$. With which, we rewrite (36) and (37) as:

$$
\begin{gathered}
f(t)=2 u(t) b(t) . \\
\left\{\begin{array}{l}
x(t)=\alpha+\beta \int_{0}^{t} e^{G(t)} d \tau \\
G(t)=\int_{0}^{t} b(s) d s
\end{array}\right.
\end{gathered}
$$

and

$$
y(t)-2 \beta e^{G(t)} b(t)=\left(\alpha+\beta \int_{0}^{t} e^{G(t)} d \tau\right) c(t), \text { for all } t \in[0, l] .
$$

Remark 3.2. Considering the Observation 3.1, of the relations (30), (31), (32) we obtain:

$$
b(t) x^{\prime}(t)+\frac{1}{2} c(t) x(t)=\frac{y(t)}{2} \text { and } x^{\prime \prime}(t)+\frac{x(t) c(t)}{2}=\frac{y(t)}{2}
$$

We illustrate via the ordinary linear differential equation

$$
x^{\prime \prime}(t)+x^{\prime}(t)+x(t)=e^{t}, x(0)=\frac{1}{3}, x^{\prime}(0)=\frac{1}{3} .
$$

We observe that $b(t)=c(t)=1, y(t)=e^{t}$ and $\operatorname{so} G(t)=t$. Using (40) we have

$$
e^{t}-\frac{2}{3} e^{t}=\frac{1}{3}+\frac{1}{3}\left(e^{t}-1\right), \forall t \in[0, l] \text {. }
$$

Therefore, the solution is $x(t)=\frac{1}{3} e^{t}$.

Example 3.2. [4] Let $D$ be a open region and bounded in the plane $X Y$, $\partial D$ smooth boundary of $D$. Given a real function $f \in \mathcal{L}^{2}$, we would like to find a function u such that

$$
\begin{aligned}
-\Delta u & =f \text { in } D \\
u & =0 \text { en } D
\end{aligned}
$$

Solution. Suppose there is a solution for our given differential equation.

Let $T(z, w)=\left(\frac{d^{2} u}{d x^{2}}\right) z+\left(\frac{d^{2} u}{d y^{2}}\right) w$. Clearly $T$ is continuous and $\operatorname{ker} T=\left\{\left(-\frac{d^{2} u}{d y^{2}}, \frac{d^{2} u}{d x^{2}}\right)\right\},\{\operatorname{ker} T\}^{\perp}=\left\{\left(\frac{d^{2} u}{d x^{2}}, \frac{d^{2} u}{d y^{2}}\right)\right\}$. Therefore one has

$$
T(1,1)=\frac{d^{2} u}{d x^{2}}+\frac{d^{2} u}{d y^{2}}=-f
$$

Moreover

$$
(1,1)=\lambda_{1}\left(-\frac{d^{2} u}{d y^{2}}, \frac{d^{2} u}{d x^{2}}\right)+\lambda_{2}\left(\frac{d^{2} u}{d x^{2}}, \frac{d^{2} u}{d y^{2}}\right)
$$

from (43) it is clear that $\lambda_{1}=\lambda_{1}(x, y), \lambda_{2}=\lambda_{2}(x, y)$. From the relations (42) and (43) one has

$$
\begin{aligned}
& -f=\lambda_{2}\left[\left(\frac{d^{2} u}{d x^{2}}\right)^{2}+\left(\frac{d^{2} u}{d y^{2}}\right)^{2}\right] \\
& -f=\lambda_{2}\left[f^{2}-2 \frac{d^{2} u}{d x^{2}}\left(-f-\frac{d^{2} u}{d x^{2}}\right)\right]
\end{aligned}
$$

from (44) one has

$$
\begin{aligned}
& \frac{d^{2} u}{d x^{2}}=-\frac{f}{2}+\frac{1}{2} \sqrt{\frac{2 f}{\tau}-f^{2}} \\
& \frac{d^{2} u}{d y^{2}}=-\frac{f}{2}-\frac{1}{2} \sqrt{\frac{2 f}{\tau}-f^{2}}
\end{aligned}
$$


where $\tau(x, y)=-\lambda_{2}(x, y)$. If $u$ is of the class $\mathcal{C}^{2}$, then from (45) we have

$$
\iint_{D}\left(\frac{d^{2} u}{d x^{2}}-\frac{d^{2} u}{d y^{2}}\right) d x d y=\iint_{D} \sqrt{\frac{2 f}{\tau}-f^{2}} d x d y
$$

Due to the Green's theorem one has that (46) imply

$$
\iint_{D} \sqrt{\frac{2 f}{\tau}-f^{2}} d x d y=0
$$

Then from (45) one has

$$
\frac{d^{2} u}{d x^{2}}=\frac{d^{2} u}{d y^{2}}=-\frac{f}{2}
$$

Example 3.3. Let us consider the problem of finding the natural frequency and vibration modes of an extended membrane on a bounded region $D$ in $\mathbb{R}^{2}$ and boundary $\partial D$. The small amplitude periodic vibrations are described by the equation

$$
\begin{aligned}
\Delta u+\lambda u & =0, \text { en } D, \lambda>0 . \\
u & =0, \text { en } \partial D .
\end{aligned}
$$

Solution. Suppose there is a solution for our given differential equation.

Following the ideas of the example 3.2 one has that for $u$ in the class $\mathcal{C}^{2}$;

$$
\frac{d^{2} u}{d x^{2}}=\frac{d^{2} v}{d y^{2}}=\frac{-\lambda u}{2}
$$

As it is known a solution of (48) is given by

$$
u(x, y)=\left(A \cos \sqrt{\frac{\lambda}{2}} x+B \sin \sqrt{\frac{\lambda}{2}} x\right)\left(\widetilde{A} \cos \sqrt{\frac{\lambda}{2}} y+\widetilde{B} \sin \sqrt{\frac{\lambda}{2}} y\right) .
$$

If one considers $D=[-L, L] \times[-L, L]$, then from the relation (49) one has that for $f(x)=A \cos \sqrt{\lambda} x+B \sin \sqrt{\lambda} x$

$$
\begin{aligned}
f(L) & =A \cos \sqrt{\frac{\lambda}{2}} L+B \sin \sqrt{\frac{\lambda}{2}} L=0 \\
f(-L) & =A \cos \sqrt{\frac{\lambda}{2}} L-B \sin \sqrt{\frac{\lambda}{2}} L=0 .
\end{aligned}
$$

From (50) and (51) one has that $A=0$. Therefore $\sqrt{\frac{\lambda}{2}}=\frac{k \pi}{L}, k \in \mathbb{N}$. Analogously in (49) one has $\widetilde{A}=0$. Thus, one has the solution

$$
u_{k}(x, y)=C_{k}\left(\sin \frac{k \pi x}{L}\right)\left(\sin \frac{k \pi y}{L}\right), k \in \mathbb{N} .
$$

Due to the superposition principle one has

$$
u(x, y)=\sum_{k=1}^{+\infty} C_{k}\left(\sin \frac{k \pi x}{L}\right)\left(\sin \frac{k \pi y}{L}\right)
$$

\section{Conclusions}

The Theorem 2.1 shows us the possible ways of writing an even number that ends in six, as the sum of two odd numbers and conditions are established so that these odd numbers are prime. As a consequence, the Corollary ?? shows us that: if a number $M$ ending in six can be expressed as the sum of two odd prime numbers, then the prime numbers that are used to decompose $M+10$ they have a relationship with the prime numbers already used in $M$.

In the Theorem 2.2, making use of a suitable linear functional $F: \mathbb{R}^{3} \rightarrow \mathbb{R}$ we obtain natural number representations of the form $(10 A+9), A \in \mathbb{N}$ in order to obtain positive integer solutions of the quadratic equation $(10 x+9)(10 y+9)=P$ where $\mathrm{P}$ is a natural number given that it ends one. And we also obtain lower bounds and upper bounds for the number $A$. 
In the Examples 3.1, 3.2, 3.3 the same technique that helped to obtain the initial results is used. Although it is true that the first three steps of the technique are the same for all the results shown, from the fourth step on, other strategies must be sought to solve the problems raised.

For future research, in Example 3.1, the given solution is only valid for some functions $c(t), b(t)$ and $y(t)$ we suggest looking for a $f(t)$ in (27) such that the ODE is solved for any $c(t), b(t)$ and $y(t)$. It is worth noting that from the equation (47) it is possible to obtain a strong solution for the proposed problem.

As a final conclusion we believe that the technique used can be used to solve problems related to number theory, some ordinary and partial differential equations as we illustrate in this article.

\section{Acknowledgments}

The author thanks CONCYTEC for partial financial support and also thanks his family for providing him a nice working environment.

\section{REFERENCES}

[1] B. M. Cerna Maguiña, Some results on natural numbers represented by quadratic polynomials in two variables, https://arxiv.org/abs/2006.08 V1[Math.NT].

[2] P. Samuel (2013). Algebraic Theory of Numbers: Translated from the French by Allan J. Silberger. Courier Corporation.

[3] V.A Trenoguin, B.M Pisarievski, T.S Sovoleva . Editorial MIR, 1987. Problemas y Ejercicios de Análisis Funcional.

[4] Charles W. Groetsch. Elements of Applicable Functional Analysis. Marcel Dekker, INC.

[5] B.M.Cerna Maguiña, Victor H. López Solís and Dik D. Lujerio Garcia. Some Results on Analysis and number theory. https://arxiv.org/abs/2102.12379.

[6] H. Iwaniec, Primes represented by quadratic polynomials in two variables, Acta Arithmetica 24(1974), 435459.

[7] Goldoni, Luca (2010) Prime Numbers and Polynomials. PhD thesis, University of Trento.

[8] B. M. Cerna Maguiña, H. F. Cerna Maguiña and H. Blas, Some results on prime numbers, Int. J. of Pure and App. Math. 118 (2018) 845 .

[9] B.M Cerna Maguiña, H. Blas, V.H. López Solis. Some Results on natural numbers represented by quadratic polynomials in two variables. IOP SCIENCE. Published Under License by IOP. Publishin LTD Journal of Physics: Conference Series, Volume 1558 XVIJ meeting of Physics 15-17 August 2018, Lima Perú. 